\title{
Copolymer Sequence Distribution in Which Monomer Unit Sequence and Its Cotacticity Both Obey Correlatively the First Order Markov Process. Applications to Poly(acrylonitrile-co-methyl methacrylate) and Poly(acrylonitrile-co-ethyl methacrylate)
}

\author{
Kunio Hisatani \\ Fundamental Research Laboratory of Natural and Synthetic Polymers, Asahi Chemical Industry Co., Ltd., \\ 11-7 Hacchonawate Takatsuki, Osaka 569, Japan
}

(Received November 13, 1996)

\begin{abstract}
KEY WORDS Copolymer Sequence Distribution / Cotacticity / Poly(acrylonitrile-co-methyl methacrylate) / Poly(acrylonitrile-co-ethyl methacrylate) $/{ }^{13} \mathrm{C}$ NMR Spectrometer / Bernoulli Statistics / Markov Statistics / Triad Sequence /
\end{abstract}

For a long time, many scientists have dealt with an analytical theory on sequence distribution of polymers. As early as 1950s and 1960s, Frisch et al., ${ }^{1}$ Coleman, ${ }^{2}$ Miller and Nielsen, ${ }^{3}$ Fordham,,${ }^{4}$ Price, ${ }^{5}$ and Miyake and Chujo $^{6}$ made much efforts to establish the sequence distribution theory based on either the meso and racemo ( $m r$-type) and or the $D$ and $L$ form ( $D L$-type) concepts. Frisch et al. ${ }^{7,8}$ analyzed stereoregular sequence of homopolymers employing $40 \mathrm{MHz}$ NMR, which was the newest NMR equipment at those days but they could hardly obtain the reliable data to describe sequential distribution because of lack of the sufficient resolution on NMR spectra.

Regarding copolymers, Bovey's proposed concept ${ }^{9}$ made as early as 1962, "monomer unit sequence with its cotacticity" in a bi-component copolymer system, in which each of the monomer units has the possibility of yielding a stereoregular homopolymer, has encouraged many authors to study the sequence distributions of many copolymers. Despite of the theoretical concept, the triad or higher sequences of a monomer unit combined with its stereoregularity had not been reported again due to the still insufficient NMR peak resolution until late of 1980s. For example, the sequence distribution for poly(acrylonitrile(AN)-co-methyl methacrylate (MMA) $)^{10-15}$ and poly(AN-co-ethyl methacrylate (EMA) $)^{10,11}$ were reported without offering the complete longer sequence information than diad with both monomer unit and its cotacticity. But very recently, Kapur and Brar have assigned ${ }^{13} \mathrm{C}$ NMR peaks to all of 20 triads of both poly(AN-co-MMA) ${ }^{16}$ and poly(ANco-EMA $)^{17}$ using by $100 \mathrm{MHz}-{ }^{1} \mathrm{H}\left\{{ }^{13} \mathrm{C}\right\}$ NMR spectrometer.

However, considering the recent remarkable progress of NMR technology, $100 \mathrm{MHz}$ instrument employed by Kapur and Brar is not sufficient enough to settle down the peak assignments of these copolymers in view of their sequence distribution statistics.

On the one hand, the author and his collaborators ${ }^{18-20}$ have proposed a general theory for the determination of sequence distribution of bi-component copolymers, assuming that the stereoregularity of the sequence is based on cotacticity (i.e., comeso and coracemo) or chirality (i.e., $D$ and $L$ form) in the copolymer. The general theory has enabled us to express copolymer sequence including stereoregularity more precisely than before, and to give more detailed information on polymerization mechanism by examining basic probability parameters of the most suitable statistics.

In the theory ${ }^{20}$ we have dealt with the cases where monomer unit sequence distribution, MSD (e.g., $\mathrm{AABABB})$ and its cotacticity distribution, CTD (e.g., $m m r m r)$ may or may not obey the same statistical model, and also MSD does or does not correlate with CTD. Here, the former case is referred to as "correlative" and the latter case as "independent." In other words, the cotacticity between the growing chain end and the penultimate unit does or does not influence the kind of monomer to be added at the end, and the monomer unit sequence does or does not effect the cotacticity between the newly added monomer unit and the penultimate unit. The statistical model is, for example, represented as [1st Markov-Bernoulli $]_{\text {ind(cor) }}$, when MSD and CTD obey independently (correlatively) to the first Markov and Bernoulli statistics, respectively.

In the present article, a statistical model [1st Markov $]-[1 \text { st Markov }]_{\text {cor }}$ has been newly established which has not been dealt with in the previous articles, ${ }^{18-20}$ since such cases might appear very often in the bi-component copolymer field.

In order to verify the systematic analytical theory ${ }^{18-20}$ including a newly derived statistical model, ${ }^{13} \mathrm{C}$ NMR data of poly(AN-co-MMA) ${ }^{16}$ and poly(AN-co-EMA) ${ }^{17}$ reported by Kapur and Brar have been analyzed. Although more precise information than those reported Kapur and Brar could be possible using up-to-date NMR equipment with higher resolution, in the present article an emphasis is placed on how the theory could successfully apply to other scientists' data.

\section{THEORETICAL BACKGROUND}

\section{Statistical Model}

Three cases of statistical models were examined in this article as shown below. Cases (1) and (2) are exactly 
based on the Markov process in which MSD and CTD do or do not correlate to each other, respectively. Case (3) proposed by Bovey is not based on the Markov process in a strict sense but has been widely used so far, then included here as a comparison. Here, MSD and CTD are each described by either Bernoulli, or 1st and 2nd Markov statistics.

\section{Case (1) Independent Statistical Model}

This case is a combination where each MSD and CTD obeys either Bernoulli, or first and second order Markov statistics independently. The number of statistics examined in the present article is nine. The molar fractions of sequences in this case are described as multiplication of MSD by CTD. The previous article should be referred to for detail. ${ }^{18}$

\section{Case (2) Correlative Statistical Model}

This case is the correlative combination case in which both of the monomer unit sequence and its cotacticity obey either Bernoulli or first order Markov statistics correlatively. The number of models used in the present article is four, containing a statistical model [1st Markov $]-[1 \text { st Markov }]_{\text {cor }}$ which has not been derived in the previous article and is described as below:

\section{(2-1) [1st Markov $]-[\text { lst Markov }]_{\text {cor }}$ Model}

In the model, the number of independent parameters is twelve, namely three out of the four parameters for each of the next equations:

$$
\begin{aligned}
& P_{(m \mathrm{~A} / m \mathrm{~A})}+P_{(r \mathrm{~A} / m \mathrm{~A})}+P_{(m \mathrm{~B} / m \mathrm{~A})}+P_{(r \mathrm{~B} / m \mathrm{~A})}=1 \\
& P_{(m \mathrm{~A} / r \mathrm{~A})}+P_{(r \mathrm{~A} / r \mathrm{AA})}+P_{(m \mathrm{~B} / \mathrm{rA})}+P_{(r \mathrm{~B} / r \mathrm{~A})}=1 \\
& P_{(m \mathrm{~A} / m \mathrm{~B})}+P_{(r \mathrm{~A} / m \mathrm{~B})}+P_{(m \mathrm{~B} / m \mathrm{~B})}+P_{(r \mathrm{~B} / m \mathrm{~B})}=1 \\
& P_{(m \mathrm{~A} / r \mathrm{~B})}+P_{(r \mathrm{~A} / r \mathrm{~B})}+P_{(m \mathrm{~B} / r \mathrm{~B})}+P_{(r \mathrm{~B} / r \mathrm{~B})}=1
\end{aligned}
$$

In the above equations, $P_{(r \mathrm{~A} / m \mathrm{~B})}$, for example, means an addition probability of monomer A with coracemo type to the growing chain end of monomer B with comeso diad.

All of the eight diad sequences with direction should satisfy the followings:

$$
\begin{aligned}
{\left[\mathrm{A}^{m} \mathrm{~A}\right]=} & {\left[\mathrm{A}^{m} \mathrm{~A}\right] P_{(m \mathrm{~A} / m \mathrm{~A})}+\left[\mathrm{A}^{r} \mathrm{~A}\right] \mathrm{P}_{(m \mathrm{~A} / r \mathrm{~A})} } \\
& +\left[\mathrm{B}^{m} \mathrm{~A}\right] P_{(m \mathrm{~A} / m \mathrm{~A})}+\left[\mathrm{B}^{r} \mathrm{~A}\right] P_{(m \mathrm{~A} / r \mathrm{~A})} \\
{\left[\mathrm{A}^{r} \mathrm{~A}\right]=} & {\left[\mathrm{A}^{m} \mathrm{~A}\right] P_{(r \mathrm{~A} / m \mathrm{~A})}+\left[\mathrm{A}^{r} \mathrm{~A}\right] P_{(r \mathrm{~A} / r \mathrm{~A})} } \\
& +\left[\mathrm{B}^{m} \mathrm{~A}\right] P_{(r \mathrm{~A} / m \mathrm{~A})}+\left[\mathrm{B}^{r} \mathrm{~A}\right] P_{(r \mathrm{~A} / r \mathrm{~A})} \\
{\left[\mathrm{A}^{m} \mathrm{~B}\right]=} & {\left[\mathrm{A}^{m} \mathrm{~A}\right] P_{(m \mathrm{~B} / m \mathrm{~A})}+\left[\mathrm{A}^{r} \mathrm{~A}\right] P_{(m \mathrm{~B} / r \mathrm{~A})} } \\
& +\left[\mathrm{B}^{m} \mathrm{~A}\right] P_{(m \mathrm{~B} / m \mathrm{~A})}+\left[\mathrm{B}^{r} \mathrm{~A}\right] P_{(m \mathrm{~B} / r \mathrm{~A})} \\
{\left[\mathrm{A}^{r} \mathrm{~B}\right]=} & {\left[\mathrm{A}^{m} \mathrm{~A}\right] P_{(r \mathrm{~B} / m \mathrm{~A})}+\left[\mathrm{A}^{r} \mathrm{~A}\right] P_{(r \mathrm{~B} / r \mathrm{~A})} } \\
& +\left[\mathrm{B}^{m} \mathrm{~A}\right] P_{(r \mathrm{~B} / m \mathrm{~A})}+\left[\mathrm{B}^{r} \mathrm{~A}\right] P_{(r \mathrm{~B} / r \mathrm{~A})} \\
{\left[\mathrm{B}^{m} \mathrm{~A}\right]=} & {\left[\mathrm{A}^{m} \mathrm{~B}\right] P_{(m \mathrm{~A} / m \mathrm{~B})}+\left[\mathrm{A}^{r} \mathrm{~B}\right] P_{(m \mathrm{~A} / r \mathrm{~B})} } \\
& +\left[\mathrm{B}^{m} \mathrm{~B}\right] P_{(m \mathrm{~A} / m \mathrm{~B})}+\left[\mathrm{B}^{r} \mathrm{~B}\right] P_{(m \mathrm{~A} / r \mathrm{~B})} \\
{\left[\mathrm{B}^{r} \mathrm{~A}\right]=} & {\left[\mathrm{A}^{m} \mathrm{~B}\right] P_{(r \mathrm{~A} / m \mathrm{~B})}+\left[\mathrm{A}^{r} \mathrm{~B}\right] P_{(r \mathrm{~A} / r \mathrm{~B})} } \\
& +\left[\mathrm{B}^{m} \mathrm{~B}\right] P_{(r \mathrm{~A} / m \mathrm{~B})}+\left[\mathrm{B}^{r} \mathrm{~B}\right] P_{(r \mathrm{~A} / r \mathrm{~B})} \\
{\left[\mathrm{B}^{m} \mathrm{~B}\right]=} & {\left[\mathrm{A}^{m} \mathrm{~B}\right] P_{(m \mathrm{~B} / m \mathrm{~B})}+\left[\mathrm{A}^{r} \mathrm{~B}\right] P_{(m \mathrm{~B} / r \mathrm{~B})} } \\
& +\left[\mathrm{B}^{m} \mathrm{~B}\right] P_{(m \mathrm{~B} / m \mathrm{~B})}+\left[\mathrm{B}^{r} \mathrm{~B}\right] P_{(m \mathrm{~B} / r \mathrm{~B})} \\
{\left[\mathrm{B}^{r} \mathrm{~B}\right]=} & {\left[\mathrm{A}^{m} \mathrm{~B}\right] P_{(r \mathrm{~B} / m \mathrm{~B})}+\left[\mathrm{A}^{r} \mathrm{~B}\right] P_{(r \mathrm{~B} / r \mathrm{~B})} }
\end{aligned}
$$

$$
\begin{gathered}
+\left[\mathrm{B}^{m} \mathrm{~B}\right] P_{(r \mathrm{~B} / m \mathrm{~B})}+\left[\mathrm{B}^{r} \mathrm{~B}\right] P_{(r \mathrm{~B} / r \mathrm{~B})} \\
{\left[\mathrm{A}^{m} \mathrm{~A}\right]+\left[\mathrm{A}^{r} \mathrm{~A}\right]+\left[\mathrm{A}^{m} \mathrm{~B}\right]+\left[\mathrm{A}^{r} \mathrm{~B}\right]+\left[\mathrm{B}^{m} \mathrm{~A}\right]} \\
+\left[\mathrm{B}^{r} \mathrm{~A}\right]+\left[\mathrm{B}^{m} \mathrm{~B}\right]+\left[\mathrm{B}^{r} \mathrm{~B}\right]=1
\end{gathered}
$$

where eq $5 \mathrm{i}$ is a normalizing condition and eight of the nine equations are independent. It is difficult to solve completely exactly these equations, but introduction of two relations, $\left[\mathrm{A}^{m} \mathrm{~B}\right]=\left[\mathrm{B}^{m} \mathrm{~A}\right]$ and $\left[\mathrm{A}^{r} \mathrm{~B}\right]=\left[\mathrm{B}^{r} \mathrm{~A}\right]$ which are appropriate assumptions in the polymer, leads to solutions of triad sequence fractions as follows:

$$
\begin{aligned}
& \left(\mathrm{A}^{m} \mathrm{~A}^{m} \mathrm{~A}\right)=P_{(m \mathrm{~A} / m \mathrm{~A})}\left(P_{(m \mathrm{~A} / m \mathrm{~A})} P_{(m / r \mathrm{~A})}\right. \\
& \left.+P_{(m \mathrm{~A} / \mathrm{rA})} P_{(r / m \mathrm{~A})}\right) D_{\{\mathrm{A} / \mathrm{B}\}} / D_{11}^{2 M^{*} m r} \\
& \left(\mathrm{~A}^{m} \mathrm{~A}^{r} \mathrm{~A}\right)=\left\{P _ { ( r \mathrm { A } / m \mathrm { A } ) } \left(P_{(m \mathrm{~A} / m \mathrm{~A})} P_{(m / r \mathrm{~A})}\right.\right. \\
& \left.+P_{(m \mathrm{~A} / r \mathrm{~A})} P_{(r / m \mathrm{~A})}\right)+P_{(m \mathrm{~A} / r \mathrm{~A})}\left(P_{(r \mathrm{~A} / m \mathrm{~A})} P_{(m / r \mathrm{~A})}\right. \\
& \left.\left.+P_{(r \mathrm{~A} / \mathrm{rA})} P_{(r / m \mathrm{~A})}\right)\right\} D_{\{\mathrm{A} / \mathrm{B}\}} / D_{11}^{2 M^{*} m r} \\
& \left(\mathrm{~A}^{r} \mathrm{~A}^{r} \mathrm{~A}\right)=P_{(r \mathrm{~A} / r \mathrm{AA})}\left(P_{(r \mathrm{~A} / m \mathrm{~A})} P_{(m / r \mathrm{~A})}\right. \\
& \left.+P_{(r \mathrm{~A} / \mathrm{rA})} P_{(r / m \mathrm{~A})}\right) D_{\{\mathrm{A} / \mathrm{B}\}} / D_{11}^{2 M^{*} m r} \\
& \left(\mathrm{~A}^{m} \mathrm{~A}^{m} \mathrm{~B}\right)=\left\{P_{(m \mathrm{~B} / m \mathrm{~A})}\left(P_{(m \mathrm{~A} / m \mathrm{~A})} P_{(m / r \mathrm{~A})}+P_{(m \mathrm{~A} / r \mathrm{~A})} P_{(r / m \mathrm{~A})}\right)\right. \\
& +P_{(m \mathrm{~A} / m \mathrm{~A})}\left(P_{(m \mathrm{~B} / m \mathrm{~A})} P_{(m / r \mathrm{~A})}\right. \\
& \left.\left.+P_{(m \mathrm{~B} / r \mathrm{~A})} P_{(r / m \mathrm{~A})}\right)\right\} D_{\{\mathrm{A} / \mathrm{B}\}} / D_{11}^{2 M^{*} m r} \\
& \left(\mathrm{~A}^{m} \mathrm{~A}^{r} \mathrm{~B}\right)=\left\{P_{(r \mathrm{~B} / m \mathrm{~A})}\left(P_{(m \mathrm{~A} / m \mathrm{~A})} P_{(m / r \mathrm{~A})}+P_{(m \mathrm{~A} / r \mathrm{~A})} P_{(r / m \mathrm{~A})}\right)\right. \\
& +P_{(m \mathrm{~A} / r \mathrm{~A})}\left(P_{(r \mathrm{~B} / m \mathrm{~A})} P_{(m / r \mathrm{~A})}\right. \\
& \left.\left.+P_{(r \mathrm{~B} / r \mathrm{~A})} P_{(r / m \mathrm{~A})}\right)\right\} D_{\{\mathrm{A} / \mathrm{B}\}} / D_{11}^{2 M^{*} m r} \\
& \left(\mathrm{~A}^{r} \mathrm{~A}^{m} \mathrm{~B}\right)=\left\{P_{(m \mathrm{~B} / r \mathrm{AA})}\left(P_{(r \mathrm{~A} / \mathrm{m} A)} P_{(m / r \mathrm{~A})}+P_{(r \mathrm{~A} / r \mathrm{~A})} P_{(r / m \mathrm{~A})}\right)\right. \\
& +P_{(r \mathrm{~A} / m \mathrm{~A})}\left(P_{(m \mathrm{~B} / m \mathrm{~A})} P_{(m / r \mathrm{~A})}\right. \\
& \left.\left.+P_{(m \mathrm{~B} / \mathrm{rA})} P_{(\mathrm{r} / m \mathrm{~A})}\right)\right\} D_{\{\mathrm{A} / \mathrm{B}\}} / D_{11}^{2 M^{*} m r} \\
& \left(\mathrm{~A}^{r} \mathrm{~A}^{r} \mathrm{~B}\right)=\left\{P_{(r \mathrm{~B} / r \mathrm{AA})}\left(P_{(r \mathrm{~A} / m \mathrm{~A})} P_{(m / r \mathrm{~A})}+P_{(r \mathrm{~A} / r \mathrm{~A})} P_{(r / m \mathrm{~A})}\right)\right. \\
& +P_{(r \mathrm{~A} / r \mathrm{~A})}\left(P_{(r \mathrm{~B} / m \mathrm{~A})} P_{(m / r \mathrm{~A})}\right. \\
& \left.\left.+P_{(r \mathrm{~B} / r \mathrm{AA})} P_{(r / m \mathrm{~A})}\right)\right\} D_{\{\mathrm{A} / \mathrm{B}\}} / D_{11}^{2 M^{*} m r} \\
& \left(\mathrm{~B}^{m} \mathrm{~A}^{m} \mathrm{~B}\right)=P_{(m \mathrm{~B} / m \mathrm{~A})}\left(P_{(m \mathrm{~B} / m \mathrm{~A})} P_{(m / r A)}\right. \\
& \left.\left.+P_{(m \mathrm{~B} / r \mathrm{~A})} P_{(r / m \mathrm{~A})}\right) D_{\{\mathrm{A} / \mathrm{B}\}}\right\} / D_{11}^{2 M^{*} m r} \\
& \left(\mathrm{~B}^{m} \mathrm{~A}^{r} \mathrm{~B}\right)=\left\{P_{(r \mathrm{~B} / m \mathrm{~A})}\left(P_{(m \mathrm{~B} / m \mathrm{~A})} P_{(m / r \mathrm{~A})}+P_{(m \mathrm{~B} / r \mathrm{~A})} P_{(r / m \mathrm{~A})}\right)\right. \\
& +P_{(m \mathrm{~B} / r \mathrm{~A})}\left(P_{(r \mathrm{~B} / m \mathrm{~A})} P_{(m / r \mathrm{~A})}\right. \\
& \left.\left.+P_{(r \mathrm{~B} / r \mathrm{~A})} P_{(r / m \mathrm{~A})}\right)\right\} D_{\{\mathrm{A} / \mathrm{B}\}} / D_{11}^{2 M^{*} m r} \\
& \left(B^{r} A^{r} B\right)=P_{(r \mathrm{~B} / r \mathrm{~A})}\left(P_{(r \mathrm{~B} / m \mathrm{~A})} P_{(m / r \mathrm{~A})}\right. \\
& \left.+P_{(r \mathrm{~B} / r \mathrm{~A})} P_{(r / m \mathrm{~A})}\right) D_{\{\mathrm{A} / \mathrm{B}\}} / D_{11}^{2 M^{*} m r} \\
& \left(\mathrm{~A}^{m} \mathrm{~B}^{m} \mathrm{~A}\right)=P_{(m \mathrm{~A} / m \mathrm{~B})}\left(P_{(m \mathrm{~A} / m \mathrm{~B})} P_{(m / r \mathrm{~B})}\right. \\
& \left.+P_{(m \mathrm{~A} / r \mathrm{~B})} P_{(r / m \mathrm{~B})}\right) D_{\{\mathrm{B} / \mathrm{A}\}} / D_{11}^{2 M^{*} m r} \\
& \left(\mathrm{~A}^{m} \mathrm{~B}^{r} \mathrm{~A}\right)=\left\{P_{(r \mathrm{~A} / m \mathrm{~B})}\left(P_{(m \mathrm{~A} / m \mathrm{~B})} P_{(m / r \mathrm{~B})}+P_{(m \mathrm{~A} / r \mathrm{~B})} P_{(r / m \mathrm{~B})}\right)\right. \\
& +P_{(m \mathrm{~A} / r \mathrm{~B})}\left(P_{(r \mathrm{~A} / m \mathrm{~B})} P_{(m / r \mathrm{~B})}\right. \\
& \left.\left.+P_{(r \mathrm{~A} / r \mathrm{~B})} P_{(r / m \mathrm{~B})}\right)\right\} D_{\{\mathrm{B} / \mathrm{A}\}} / D_{11}^{2 M^{*} m r} \\
& \left.+P_{(r \mathrm{~A} / r \mathrm{~B})} P_{(r / m \mathrm{~B})}\right) D_{\{\mathrm{B} / \mathrm{A}\}} / D_{11}^{2 M^{*} m r} \\
& +P_{(m \mathrm{~A} / m \mathrm{~B})}\left(P_{(m \mathrm{~B} / m \mathrm{~B})} P_{(m / \mathrm{r} \mathrm{B})}\right. \\
& \left.\left.+P_{(m \mathrm{~B} / r \mathrm{~B})} P_{(r / m \mathrm{~B})}\right)\right\} D_{\{\mathrm{B} / \mathrm{A}\}} / D_{11}^{2 M^{*} m r}
\end{aligned}
$$




$$
\begin{aligned}
& \left.\left.+P_{(r \mathrm{~B} / r \mathrm{~B})} P_{(r / m \mathrm{~B})}\right)\right\} D_{\{\mathrm{B} / \mathrm{A}\}} / D_{11}^{2 M^{*} m r} \\
\left(\mathrm{~A}^{r} \mathrm{~B}^{m} \mathrm{~B}\right)= & \left\{P_{(m \mathrm{~B} / \mathrm{r})}\left(P_{(r \mathrm{~A} / m \mathrm{~B})} P_{(m / r \mathrm{~B})}+P_{(r \mathrm{~A} / r \mathrm{~B})} P_{(r / m \mathrm{~B})}\right)\right. \\
& +P_{(r \mathrm{~A} / m \mathrm{~B})}\left(P_{(m \mathrm{~B} / m \mathrm{~B})} P_{(m / r \mathrm{~B})}\right. \\
& \left.\left.+P_{(m \mathrm{~B} / r \mathrm{~B})} P_{(r / m \mathrm{~B})}\right)\right\} D_{\{\mathrm{B} / \mathrm{A}\}} / D_{11}^{2 M^{*} m r} \\
\left(\mathrm{~A}^{r} \mathrm{~B}^{r} \mathrm{~B}\right)= & \left\{P_{(r \mathrm{~B} / r \mathrm{~B})}\left(P_{(r \mathrm{~A} / m \mathrm{~B})} P_{(m / r \mathrm{~B})}+P_{(r \mathrm{~A} / r \mathrm{~B})} P_{(r / m \mathrm{~B})}\right)\right. \\
& +P_{(r \mathrm{~A} / r \mathrm{~B})}\left(P_{(r \mathrm{~B} / m \mathrm{~B})} P_{(m / r \mathrm{~B})}\right. \\
& \left.\left.+P_{(r \mathrm{~B} / \mathrm{B} \mathrm{B})} P_{(r / m \mathrm{~B})}\right)\right\} D_{\{\mathrm{B} / \mathrm{A}\}} D_{11}^{2 M^{*} m r} \\
\left(\mathrm{~B}^{m} \mathrm{~B}^{m} \mathrm{~B}\right)= & P_{(m \mathrm{~B} / m \mathrm{~B})}\left(P_{(m \mathrm{~B} / m \mathrm{~B})} P_{(m / r \mathrm{~B})}\right. \\
& \left.+P_{(m \mathrm{~B} / r \mathrm{~B})} P_{(r / m \mathrm{~B})}\right) D_{\{\mathrm{B} / \mathrm{A}\}} D_{11}^{2 M^{*} m r} \\
\left(\mathrm{~B}^{m} \mathrm{~B}^{r} \mathrm{~B}\right)= & \left\{P_{(r \mathrm{~B} / m \mathrm{~B})}\left(P_{(m \mathrm{~B} / m \mathrm{~B})} P_{(m / r \mathrm{~B})}+P_{(m \mathrm{~B} / r \mathrm{~B})} P_{(r / m \mathrm{~B})}\right)\right. \\
& +P_{(m \mathrm{~B} / r \mathrm{~B})}\left(P_{(r \mathrm{~B} / m \mathrm{~B})} P_{(m / \mathrm{r})}\right. \\
& \left.\left.\left.+P_{(r \mathrm{~B} / \mathrm{B} \mathrm{B})} P_{(r / m \mathrm{~B})}\right)\right\} D_{\{\mathrm{B} / \mathrm{A}\}}\right\} D_{11}^{2 M^{*} m r} \\
\left(\mathrm{~B}^{r} \mathrm{~B}^{r} \mathrm{~B}\right)= & P_{(r \mathrm{~B} / \mathrm{r})}\left(P_{(r \mathrm{~B} / m \mathrm{~B})} P_{(m / r \mathrm{~B})}\right. \\
& \left.+P_{(r \mathrm{~B} / \mathrm{B})} P_{(r / m \mathrm{~B})}\right) D_{\{\mathrm{B} / \mathrm{A}\}} / D_{11}^{2 M^{*} m r}
\end{aligned}
$$

where $D_{\{\mathbf{A} / \mathbf{B}\}}, D_{\{\mathbf{B} / \mathbf{A}\}}$ and $D_{11}^{2 M^{*} m r}$ mean:

$$
\begin{aligned}
D_{\{\mathrm{A} / \mathrm{B}\}}= & P_{(\mathrm{A} / m \mathrm{~B})} P_{(m / r \mathrm{~B})}+P_{(\mathrm{A} / r \mathrm{~B})} P_{(r / m \mathrm{~B})} \\
D_{\{\mathrm{B} / \mathrm{A}\}}= & P_{(\mathrm{B} / r \mathrm{~A})} P_{(r / m \mathrm{~A})}+P_{(\mathrm{B} / m \mathrm{~A})} P_{(m / r \mathrm{~A})} \\
D_{11}^{2 M^{*} m r}= & \left(P_{(r / m \mathrm{~A})}+P_{(m / r \mathrm{~A})}\right) D_{\{\mathrm{A} / \mathrm{B}\}}+\left(P_{(r / m \mathrm{~B})}\right. \\
& \left.+P_{(m / r \mathrm{~B})}\right) D_{\{\mathrm{B} / \mathrm{A}\}}
\end{aligned}
$$

\section{Case (3) $\sigma$-Parameter Model}

In this article, a statistical model including cotacticity parameters $\sigma_{\mathrm{AA}}, \sigma_{\mathrm{AB}}, \sigma_{\mathrm{BA}}$, and $\sigma_{\mathrm{BB}}$ proposed by Bovey ${ }^{1}$ has been employed in order to compare with the Markov statistical models shown above. Here, a parameter $\sigma_{\mathrm{AB}}$, for example, is referred to as the probability of comeso arrangement in an A-B diad sequence. This statistics is a case in which both MSD and CTD correlate to each other, and the former is dealt as almost the first order Markov statistics and the latter as Bernoulli. Since cotacticity in the model depends only on the monomer unit diad sequence, the model corresponds to [1st Markov $]-[\text { Bernoulli }]_{\text {cor }}$ in case (2).

\section{The Selection of the Most Suitable Model}

The method to select the most suitable statistics is as same as that already described in ref 19 and 20, in which $\delta_{\min }^{\prime}$, the minimum deviation between calculated and theoretical fractions, will be estimated.

\section{RESULTS AND DISCUSSION}

\section{Poly(acrylonitrile-co-methyl methacrylate)}

Table I cites the triad molar fraction data analyzed from ${ }^{13} \mathrm{C}$ NMR spectra of carbonyl and nitrile carbon of poly (AN-co-MMA) reported by Kapur and Brar, ${ }^{16}$ which were normalized over whole copolymer fractions to be ready for statistical calculations, because the original paper contained those normalized only for each carbonyl or nitrile carbon. These polymers were synthesized by radical bulk method. Hereafter AN and MMA units are shortly referred to as $\mathrm{A}$ and $\mathrm{M}$, respectively.

Based on the triad fractions in the Table I, the value of $\delta_{\min }^{\prime} \times 1000$ defined in eq 20 for each copolymer was estimated and summarized in Table II. The table tells that the most suitable statistical model is [1st Markov][1st Markov $]_{\text {cor }}$ for each sample.

Table III gathers the values of the addition probability parameters obtained in the most suitable statistical model. If we want to know whether stereospecific polymerization does or does not go, we may compare the values of parameters $P_{(m \mathrm{X} / \mathrm{End})}$ and $P_{(r \mathrm{X} / \mathrm{End})}$. The former means a probability for monomer $\mathrm{X}$ to add with comesotype to the propagating end (End), and the latter with coracemo-type. Table III says the above addition probabilities are at almost the same level within each sample $(0.217-0.247$ for $79.0 \% \mathrm{~A}$ and $0.106-0.115$ for $59.6 \% \mathrm{~A}$ )

\begin{tabular}{|c|c|c|c|c|c|}
\hline \multicolumn{3}{|c|}{$\mathrm{CN}$ carbon } & \multicolumn{3}{|c|}{ Co carbon } \\
\hline \multirow{2}{*}{ Triad sequence } & \multicolumn{2}{|c|}{ Observed mol fraction } & \multirow{2}{*}{ Triad sequence } & \multicolumn{2}{|c|}{ Observed mol fraction } \\
\hline & $79.0 \% \mathrm{~A}$ & $59.6 \% \mathrm{~A}$ & & $79.0 \% \mathrm{~A}$ & $59.6 \% \mathrm{~A}$ \\
\hline $\mathrm{A}^{r} \mathrm{~A}^{r} \mathrm{~A}^{\mathrm{a}}$ & 0.0616 & - & $\mathrm{A}^{m} \mathrm{~A}^{m} \mathrm{~A}^{\mathrm{b}}$ & 0.0599 & 0.0533 \\
\hline $\mathrm{A}^{m} \mathrm{~A}^{r} \mathrm{~A}$ & $0.0776 ́$ & - & $\mathrm{A}^{m} \mathrm{M}^{r} \mathrm{~A}$ & 0.0960 & 0.0803 \\
\hline $\mathrm{A}^{m} \mathrm{~A}^{m} \mathrm{~A}$ & 0.0333 & - & $\mathrm{A}^{r} \mathrm{M}^{r} \mathrm{~A}$ & 0.0449 & 0.0270 \\
\hline $\mathrm{A}^{r} \mathrm{~A}^{r} \mathrm{M}$ & 0.0659 & 0.0297 & $\mathrm{~A}^{m} \mathrm{M}^{m} \mathrm{M}$ & 0.0165 & 0.0311 \\
\hline $\mathrm{A}^{r} \mathrm{~A}^{m} \mathrm{M}$ & 0.0764 & 0.0397 & $\mathrm{~A}^{m} \mathrm{M}^{r} \mathrm{M}$ & 0.0618 & 0.1324 \\
\hline $\mathrm{A}^{m} \mathrm{~A}^{r} \mathrm{M}$ & 0.0561 & 0.0301 & $\mathrm{~A}^{r} \mathrm{M}^{m} \mathrm{M}$ & 0.0219 & 0.0539 \\
\hline $\mathrm{A}^{m} \mathrm{~A}^{m} \mathrm{M}$ & 0.0671 & 0.0385 & $\mathrm{~A}^{r} \mathrm{M}^{r} \mathrm{M}$ & 0.0572 & 0.1006 \\
\hline $\mathbf{M}^{r} \mathbf{A}^{r} \mathbf{M}$ & 0.0400 & 0.0445 & $\mathbf{M}^{m} \mathbf{M}^{r} \mathbf{M}$ & - & 0.0384 \\
\hline $\mathrm{M}^{m} \mathrm{~A}^{r} \mathrm{M}$ & 0.0862 & 0.1131 & $\mathrm{M}^{r} \mathbf{M}^{r} \mathrm{M}$ & - & 0.0767 \\
\hline $\mathbf{M}^{m} \mathrm{~A}^{m} \mathrm{M}$ & 0.0493 & 0.0786 & $\mathbf{M}^{m} \mathbf{M}^{m} \mathbf{M}$ & - & 0.0599 \\
\hline
\end{tabular}
in the case where a monomer $\mathrm{AN}$ adds to a propagating end of AN unit (hereafter, referred to as AN-end). Then, it seems that stereoregular polymerization does not occur. This phenomenon is considered to be similar with that of completely atactic radical homopolymerization of AN reported by many scientists including Yamazaki et $a .^{20,21}$

In the case that monomer MMA adds to $\mathrm{AN}$-end, the probability ratios of comeso and coracemo addition are $0.303 / 0.233=1.300$ and $0.255 / 0.187=1.364$ for $79.0 \% \mathrm{~A}$, and $0.451 / 0.327=1.379$ and $0.431 / 0.352=1.244$ for $59.8 \% \mathrm{~A}$. These values show that comeso addition is slightly more dominant than coracemo one.

Similarly, when monomer AN adds to MMA-end because comeso-type addition occurs slightly more fre-

Table I. Molar fractions of triads with cotacticity for poly(acrylonitrile(A)-co-methyl methacrylate(M)) samples cited from ref 17

a ${ }^{\text {, Coracemo. }}{ }^{\mathrm{b} m}$, Comeso. 
Table II. $\delta_{\min }^{\prime} \times 1000^{\text {a }}$ evaluated from various statistics on poly(acrylonitrile(A)-co-methyl methacrylate(M)) samples

\begin{tabular}{|c|c|c|c|c|c|c|c|c|}
\hline \multirow{5}{*}{ Sample code } & \multicolumn{8}{|c|}{$\delta_{\min }^{\prime} \times 1000$} \\
\hline & \multicolumn{7}{|c|}{ General statistical model } & \multirow{4}{*}{$\begin{array}{c}\begin{array}{c}\sigma \text {-Parameter } \\
\text { model }\end{array} \\
\text { Correlative } \\
\text { MSD } \\
\text { 1st Markov }\end{array}$} \\
\hline & \multicolumn{4}{|c|}{ Independent } & \multicolumn{3}{|c|}{ Correlative } & \\
\hline & \multirow{2}{*}{$\mathrm{CTD}^{\mathrm{c}}$} & \multicolumn{3}{|c|}{$\mathrm{MSD}^{\mathrm{b}}$} & \multirow{2}{*}{ CTD } & \multicolumn{2}{|c|}{ MSD } & \\
\hline & & Bernoulli & $1 \mathrm{st}^{\mathrm{d}}$ Markov & 2nd ${ }^{\mathrm{e}}$ Markov & & Bernoulli & 1st Markov & \\
\hline \multirow{3}{*}{$79.0 \% \mathrm{~A}$} & Bernoulli & 4.76 & 3.07 & 2.73 & Bernoulli & 4.76 & 2.02 & 1.95 \\
\hline & 1st Markov & 4.74 & 3.05 & 2.71 & 1st Mariov & 4.74 & $1.67^{f}$ & \\
\hline & 2nd Markov & 4.74 & 3.05 & 2.71 & & & & \\
\hline \multirow[b]{2}{*}{$59.6 \% \mathrm{~A}$} & Bernoulli & 6.44 & 4.47 & 4.34 & Bernoulli & 5.88 & 1.60 & 1.58 \\
\hline & 1st Markov & 6.30 & 4.40 & 4.21 & 1st Markov & 5.49 & $\underline{1.32}$ & \\
\hline
\end{tabular}

${ }^{a} \delta_{\min }^{\prime}$, minimized value of $\delta^{\prime} .{ }^{\mathrm{b}} \mathrm{MSD}$, monomer unit sequence distribution. ${ }^{\mathrm{c}} \mathrm{CTD}$, stereoregularity distribution. ${ }^{\mathrm{d}} 1 \mathrm{st}$ Markov, 1 st order Markov statistics. ${ }^{\mathrm{e}}$ 2nd Markov, 2nd order Markov statistics. ${ }^{\mathrm{f}}$ Underline, the most suitable statistics for each sample.

Table III. Statistical parameters for the most suitable statistical model [1st Markov]-[1st Markov $]_{\text {cor }}$ on poly(acrylonitrile(A)-co-methyl methacrylate(M)) samples

\begin{tabular}{|c|c|c|c|c|c|}
\hline \multirow{2}{*}{ Parameter } & \multicolumn{2}{|c|}{ Sample } & \multirow{2}{*}{ Parameter } & \multicolumn{2}{|c|}{ Sample } \\
\hline & $79.0 \% \mathrm{~A}$ & $59.6 \% \mathrm{~A}$ & & $79.0 \% \mathrm{~A}$ & $59.6 \% \mathrm{~A}$ \\
\hline$P_{(r \mathrm{~A} / m \mathrm{~A})}$ & 0.247 & 0.115 & $P_{(r \mathrm{~A} / m \mathrm{M})}$ & 0.320 & 0.269 \\
\hline$P_{(m \mathrm{~A} / m \mathrm{~A})}$ & 0.303 & 0.451 & $P_{(m \mathrm{M} / m \mathrm{M})}$ & 0.056 & 0.075 \\
\hline$P_{(r \mathrm{M} / m \mathrm{~A})}$ & 0.233 & 0.327 & $P_{(r \mathrm{M} / m \mathrm{M})}$ & 0.207 & 0.356 \\
\hline$P_{(m \mathrm{~A} / r \mathrm{~A})}$ & 0.230 & 0.111 & $P_{(m \mathrm{~A} / r \mathrm{M})}$ & 0.402 & 0.285 \\
\hline$P_{(r \mathrm{~A} / r \mathrm{~A})}$ & 0.328 & 0.106 & $P_{(r \mathrm{~A} / r \mathrm{M})}$ & 0.343 & 0.214 \\
\hline$P_{(m \mathrm{M} / r \mathrm{~A})}$ & 0.255 & 0.431 & $P_{(m \mathrm{M} / r \mathrm{M})}$ & 0.078 & 0.142 \\
\hline$P_{(r \mathrm{M} / r \mathrm{~A})}$ & 0.187 & 0.352 & $P_{(r \mathrm{M} / r \mathrm{M})}$ & 0.177 & 0.359 \\
\hline
\end{tabular}
unit A.

${ }^{\text {a }} P_{(m \mathrm{~A} / \mathrm{mA})}$, for example, the probability for the comeso addition of monomer unit A to the growing comeso chain end consisting of monomer

Table IV Molar fractions of triads with cotacticity for poly(acrylonitrile(A)-co-ethyl methacrylate(E)) samples cited from ref 18

\begin{tabular}{|c|c|c|c|c|c|c|c|}
\hline \multicolumn{4}{|c|}{$\mathrm{CN}$ carbon } & \multicolumn{4}{|c|}{$\mathrm{CO}$ carbon } \\
\hline \multirow{2}{*}{ Triad sequence } & \multicolumn{3}{|c|}{ Observed mol fraction } & \multirow{2}{*}{ Triad sequence } & \multicolumn{3}{|c|}{ Observed mol fraction } \\
\hline & $90.0 \% \mathrm{~A}$ & $79.8 \% \mathrm{~A}$ & $70.0 \% \mathrm{~A}$ & & $90.0 \% \mathrm{~A}$ & $79.8 \% \mathrm{~A}$ & $70.0 \% \mathrm{~A}$ \\
\hline $\mathrm{A}^{r} \mathrm{~A}^{r} \mathrm{~A}^{\mathrm{a}}$ & 0.0613 & - & - & $\mathrm{A}^{m} \mathrm{E}^{m} \mathrm{~A}^{\mathrm{b}}$ & 0.0462 & 0.0508 & 0.0512 \\
\hline $\mathrm{A}^{m} \mathrm{~A}^{r} \mathrm{~A}$ & 0.1351 & 0.0691 & - & $\mathrm{A}^{m} \mathrm{E}^{r} \mathrm{~A}$ & 0.1010 & 0.1102 & 0.1183 \\
\hline $\mathrm{A}^{r} \mathrm{~A}^{r} \mathrm{E}$ & 0.0913 & 0.0572 & 0.0355 & $\mathrm{~A}^{m} \mathrm{E}^{m} \mathrm{E}$ & 0.0070 & 0.0200 & 0.0256 \\
\hline $\mathrm{A}^{r} \mathrm{~A}^{m} \mathrm{E}$ & 0.0898 & 0.0781 & 0.0350 & $A^{m} E^{r} E$ & 0.0243 & 0.0551 & 0.0916 \\
\hline $\mathrm{A}^{m} \mathrm{~A}^{r} \mathrm{E}$ & 0.0840 & 0.0753 & 0.0464 & $\mathrm{~A}^{r} \mathrm{E}^{m} \mathrm{E}$ & 0.0081 & 0.0217 & 0.0278 \\
\hline$A^{m} A^{m} E$ & 0.0876 & 0.0600 & 0.0655 & $\mathrm{~A}^{r} \mathrm{E}^{r} \mathrm{E}$ & 0.0238 & 0.0712 & 0.1014 \\
\hline $\mathrm{E}^{r} \mathrm{~A}^{r} \mathrm{E}$ & - & 0.0572 & 0.0382 & $\mathrm{E}^{m} \mathrm{E}^{m} \mathrm{E}$ & - & 一 & 0.0093 \\
\hline $\mathrm{E}^{m} \mathrm{~A}^{r} \mathrm{E}$ & 0.0832 & 0.0979 & 0.1183 & $\mathrm{E}^{m} \mathrm{E}^{r} \mathrm{E}$ & - & - & 0.0218 \\
\hline $\mathrm{E}^{m} \mathrm{~A}^{m} \mathrm{E}$ & 0.0336 & 0.0436 & 0.0810 & $E^{r} E^{r} E$ & - & - & 0.0458 \\
\hline
\end{tabular}

${ }^{\text {a }}$, Coracemo. ${ }^{\mathrm{b} m}$, Comeso.

quently than coracemo, the addition parameter ratios are 1.303 and 1.172 for $79.0 \% \mathrm{~A}$, and 1.115 and 1.332 for $59.8 \% \mathrm{~A}$. In short, if the one of the propagating end with a monomer unit is added by the different monomer specie, comeso addition occurs relatively more often than coracemo one.

To the contrary, however, monomer MMA adds to MMA-end preferentially in coracemo arrangement, which phenomenon has been well-known for long years in MMA homopolymer ${ }^{15}$ and was reported by Kapur and Brar for copolymers. ${ }^{16}$ The ratio of coracemo addition to comeso one ranges $4-5$ times for comeso MMA-end and about 2.5 for coracemo MMA-end.

Poly(acrylonitrile-co-ethyl methacrylate)

Table IV summarizes the triad data of three poly(AN- 
Table V. $\quad \delta_{\min }^{\prime} \times 1000^{\text {a }}$ evaluated from various statistics on poly(acrylonitrile(A)-co-ethyl methacrylate(M)) samples

\begin{tabular}{|c|c|c|c|c|c|c|c|c|}
\hline \multirow{5}{*}{ Sample code } & \multicolumn{8}{|c|}{$\delta_{\min }^{\prime} \times 1000$} \\
\hline & \multicolumn{7}{|c|}{ General statistical model } & \multirow{4}{*}{$\begin{array}{c}\begin{array}{c}\sigma \text {-Parameter } \\
\text { model }\end{array} \\
\text { Correlative } \\
\text { MSD } \\
1 \text { st Markov }\end{array}$} \\
\hline & \multirow{3}{*}{$\mathrm{CTD}^{\mathrm{c}}$} & \multicolumn{3}{|c|}{ Independent } & \multicolumn{3}{|c|}{ Correlative } & \\
\hline & & \multicolumn{3}{|c|}{$\mathrm{MSD}^{\mathrm{b}}$} & \multirow{2}{*}{ CTD } & \multicolumn{2}{|c|}{ MSD } & \\
\hline & & Bernoulli & $1 s \mathrm{~d}^{\mathrm{d}}$ Markov & 2nd ${ }^{\mathrm{e}}$ Markov & & Bernoulli & 1st Markov & \\
\hline \multirow{3}{*}{$90.0 \% \mathrm{~A}$} & Bernoulli & 4.37 & 2.55 & 2.53 & Bernoulli & 4.35 & 2.01 & \multirow[t]{3}{*}{1.94} \\
\hline & 1st Markov & 4.34 & 2.52 & 2.52 & 1st Markov & 4.28 & $1.15^{\mathrm{f}}$ & \\
\hline & 2nd Markov & 4.34 & 2.52 & 2.52 & & & & \\
\hline \multirow{3}{*}{$79.8 \% \mathrm{~A}$} & Bernoulli & 5.94 & 3.02 & 2.85 & Bernoulli & 5.58 & 2.19 & \multirow{3}{*}{2.14} \\
\hline & Ist Markov & 5.88 & 3.02 & 2.82 & 1st Markov & 5.47 & 1.24 & \\
\hline & 2nd Markov & 5.88 & 3.02 & 2.82 & & & & \\
\hline \multirow{3}{*}{$70.0 \% \mathrm{~A}$} & Bernoulli & 7.60 & 4.47 & 4.37 & Bernoulli & 6.60 & 1.89 & \multirow[t]{3}{*}{1.87} \\
\hline & 1st Markov & 7.59 & 4.46 & 4.36 & 1st Markov & 5.87 & 1.08 & \\
\hline & 2nd Markov & 7.59 & 4.46 & 4.36 & & & & \\
\hline
\end{tabular}

${ }^{\mathrm{a}} \delta_{\min }^{\prime}$, minimized value of $\delta^{\prime} .{ }^{\mathrm{b}} \mathrm{MSD}$, monomer unit sequence distribution. ${ }^{\mathrm{c}} \mathrm{CTD}$, stereoregularity distribution. ${ }^{\mathrm{d}} 1 \mathrm{st}$ Markov, 1 st order Markov statistics. ${ }^{\mathrm{e}}$ 2nd Markov, 2nd order Markov statistics. ${ }^{\mathrm{f}}$ Underline, the most suitable statistics for each sample.

Table VI. Statistical parameters for the most suitable statistical model [1st Markov]-[1st Markov $]_{\text {cor }}$ on poly(acrylonitrile(A)-co-ethyl methacrylate(E)) samples

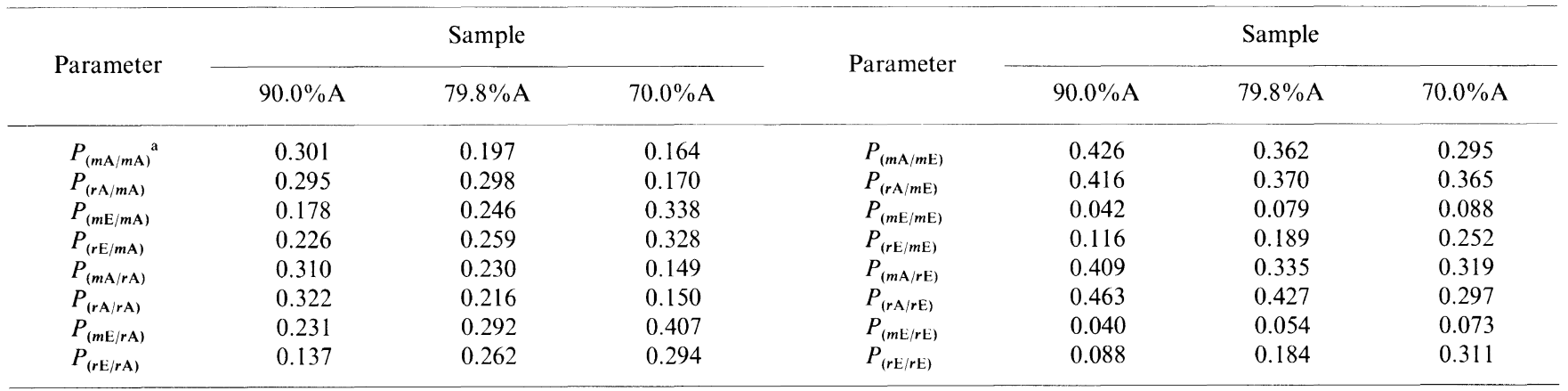

${ }^{a} P_{(m \mathrm{~A} / m \mathrm{~A})}$, for example, the probability for the comeso addition of monomer unit A to the growing comeso chain end consisting of monomer unit A.

Table VII. Diad stereoregularity for each combination of propagating end unit and additive monomer

\begin{tabular}{|c|c|c|c|}
\hline Copolymer & $\begin{array}{l}\text { Propagating } \\
\text { end unit }\end{array}$ & $\begin{array}{l}\text { Additive } \\
\text { monomer }\end{array}$ & $\begin{array}{c}\text { Diad } \\
\text { stereoregularity }\end{array}$ \\
\hline \multirow{4}{*}{ Poly(AN-co-MMA) } & $\mathrm{AN}$ & $\mathrm{AN}$ & Atactic \\
\hline & $\mathrm{AN}$ & MMA & Slightly isotactic \\
\hline & MMA & AN & Slightly isotactic \\
\hline & MMA & MMA & Syndiotactic \\
\hline \multirow{4}{*}{ Poly(AN-co-EMA) } & $\mathrm{AN}$ & $\mathrm{AN}$ & Atactic \\
\hline & $\mathrm{AN}$ & EMA & Atactic \\
\hline & EMA & $\mathrm{AN}$ & Atactic \\
\hline & EMA & EMA & Syndiotactic \\
\hline
\end{tabular}

co-EMA) samples reported also by Kapur and Brar. ${ }^{17}$ Hereafter, EMA units will be shortly referred to as E.

The degree of agreement with the copolymer samples were also examined, and the values of $\delta_{\text {min }}^{\prime} \times 1000$ was shown in Table V. The table suggests that the most suitable statistical models for all three samples are [1st Markov]-[1st Markov $]_{\text {cor }}$.

Table VI collects the values of the addition probability parameters obtained in the most suitable statistical

Polym. J., Vol. 29, No. 4, 1997 model. Judging from the addition probability ratio, AN monomer adds to a propagating end of AN unit without any stereoregular preference $(0.295-0.322$ for $90.0 \% \mathrm{~A}, 0.197-0.298$ for $79.8 \% \mathrm{~A}$, and $0.149-0.170$ for $70.0 \% \mathrm{~A}$ ), showing that the stereoregular polymerization may not proceed, too.

In the case where monomer AN and EMA add to EMA- and AN-end, respectively, the probability ratios of comeso and coracemo addition for three samples do not have any significant stereospecific tendency as same as homogeneous AN diad formation. This is from the reversed case for poly(AN-co-MMA).

It was reconfirmed that monomer EMA adds to EMA-end preferentially in coracemo addition reported by Kapur and Brar. ${ }^{17}$ The ratio of coracemo addition to comeso one is 3 times for comeso EMA-end and about 2-4 for coracemo EMA-end.

\section{Copolymerization Mechanism}

Table VII summarizes the stereoregular situations to form copolymer diads for copolymerization of acrylate monomer with AN. The cases for AN and acrylate monomer to add to AN- and acrylate ends, respectively, are in such same situations as those of their atactic and 

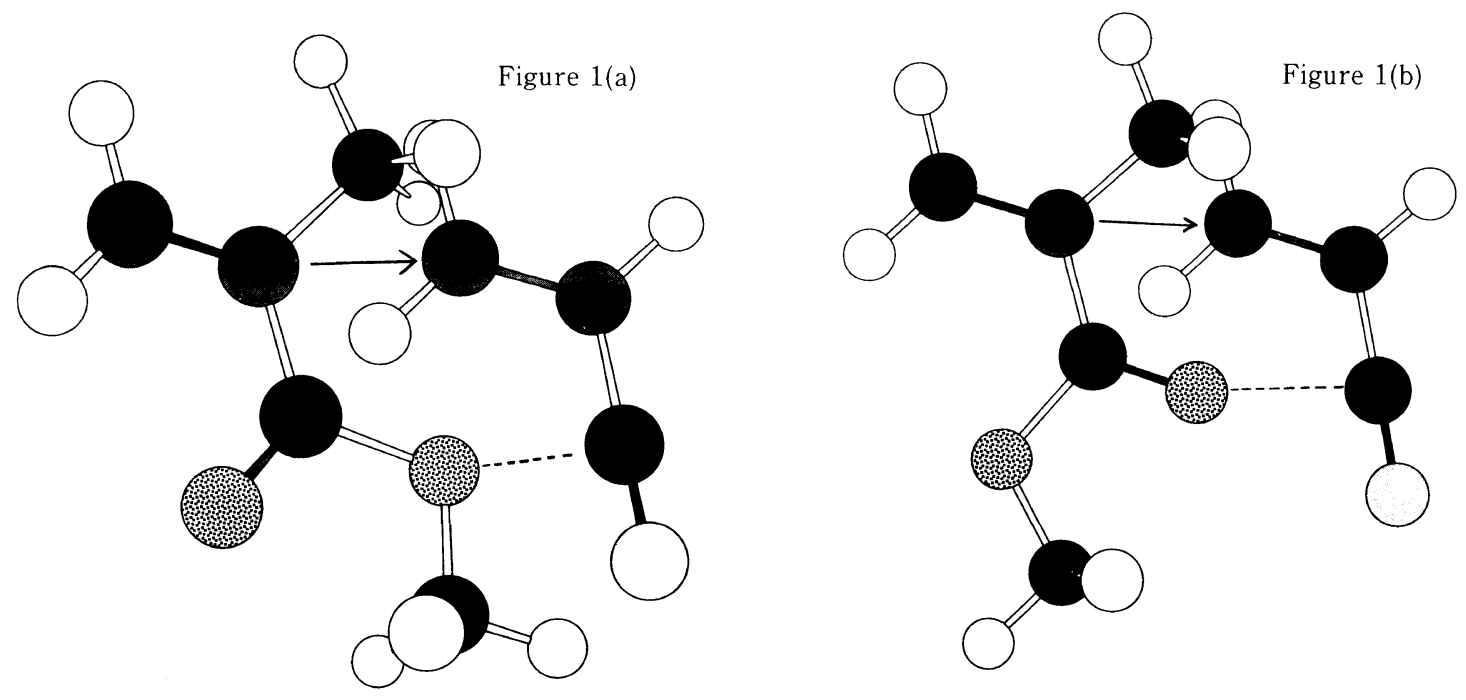

Figure 1 Interaction structures between propagating end of MMA unit and AN monomer with (a) methoxy and (b) carbonyl oxygen. Open circle, hydrogen; dotted circle, oxygen; dark curcle, carbon; light dark circle, nitrogen.
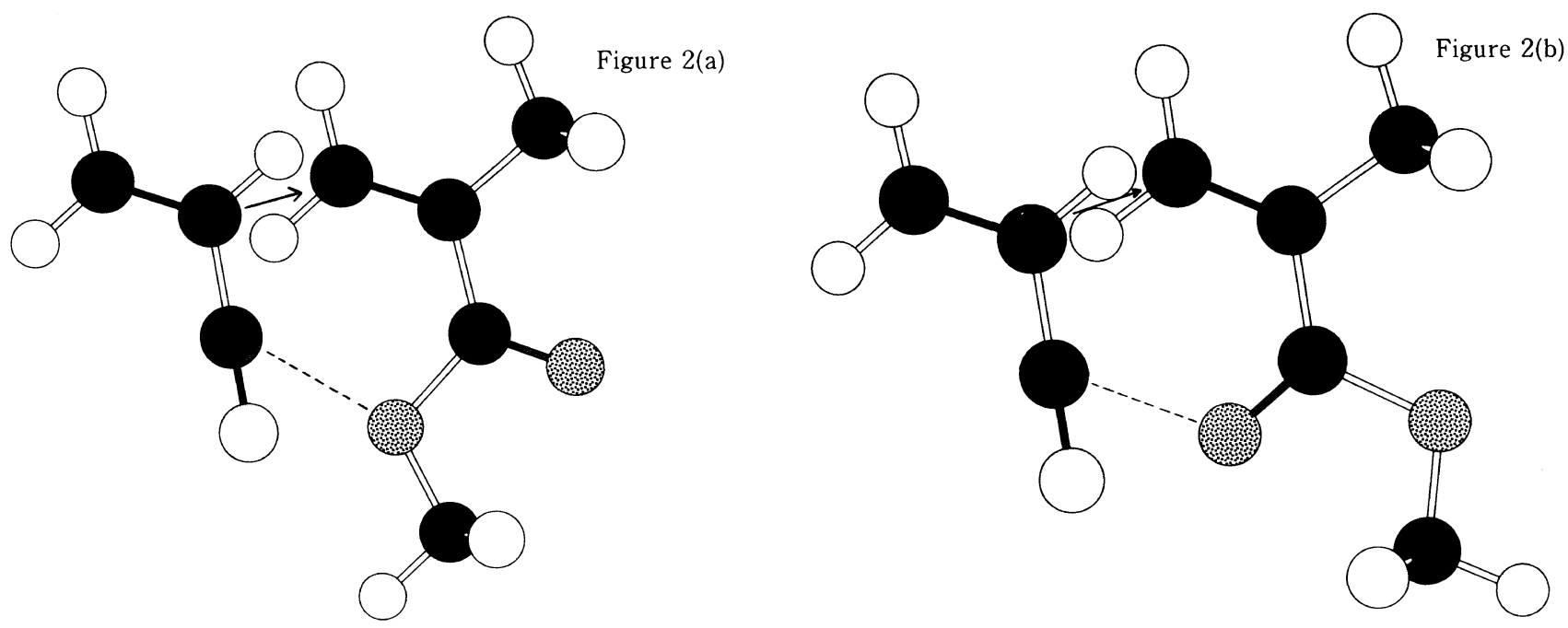

Figure 2. Interaction structures between propagating end of AN uniti and MMA monomer with (a) methoxy and (b) carbonyl oxygen.

syndiotactic homopolymerization. As for copolymerization of acrylate monomer, it is reported by Kapur and $\mathrm{Brar}^{16}$ that the probabilities to form coracemo diad are also 0.79 in MMM and EEE triads.

There, however, exists a different stereoregular tendency between MMA and EMA copolymerization with AN in the formation of heterogeneous diad parts. MMA forms the diad with AN in slightly isotactic preferential manner, but EMA-AN diad almost atactic. The above difference should be sought in the difference in the interaction form between neighboring two heterogeneous monomer units. In this connection, the interaction form of meso diad of PAN, proposed originally by Okajima et al. ${ }^{20}$ supported by some experiments (by Kamide et al. ${ }^{22-24}$ ) and by computational chemistry by Hisatani et al., ${ }^{25}$ should be taken into consideration. In the interaction form, two AN monomers forms 6-membered ring with double bond nature of nitrile groups. Similar interaction might be realized between An and MMA or EMA, the possible interaction forms are shown in Figures 1 and 2, where MMA (1) and EMA (2) adds to AN monomer. The comeso conformation of the new diad (formation of 6-membered ring like AN-AN diad) might be possible rather for $\mathrm{AN}-$ MMA than AN-EMA, probably due to the difference in bulkiness of side chains of MMA and EMA.

Acknowledgment. The author would like to express his appreciation to Dr. K. Okajima (Asahi Chemical Ind. Co., Ltd.) for his invaluable suggestions and helpful discussions.

\section{REFERENCES}

1. H. L. Frisch, C. Shuerch, and M. Szwarc, J. Polym. Sci., 11, 559 (1953).

2. B. D. Coleman, J. Polym. Sci., 31, 155 (1958).

3. R. L. Miller and L. E. Nielsen, J. Polym. Sci., 46, 303 (1960).

4. J. W. L. Fordham, J. Polym. Sci., 39, 321 (1959).

5. F. Price, J. Chem. Phys., 36, 209 (1962).

6. A. Miyake and R. Chujo, J. Polym. Sci., 46, 163 (1960).

7. H. L. Frisch, C. L. Mallows, and F. A. Bovey, J. Chem. Phys., 45, 1565 (1966)

8. H. L. Frisch, C. L. Mallows, F. Heatley, and F. A. Bovey, Macromolecules, 6, 533 (1968).

9. F. A. Bovey, J. Polym. Sci., 62, 197 (1962).

10. J. Guillot, A. Guyot, and Q. T. Pham, J. Macromol. Sci. A, 2, 1303 (1968). 
11. A. Guyot and J. Guillot, J. Macromol. Sci., 2, 889 (1968).

12. A. Guyot, Ann. Chim., 3, 441 (1968).

13. J. Cattiaux, T. Suzuki, and H. J. Harwood, J. Appl. Polym. Sci., Appl. Polym. Symp., 34, 1 (1978).

14. Q. T. Pham, Nucl. Magn. Reson., 4, 119 (1971).

15. T. A. Gerken and W. M. Ritchey, J. Appl. Polym. Sci., Appl. Polym. Symp., 34, 17 (1978).

16. G. S. Kapur and A. S. Brar, Polymer, 32, $1112(1991)$

17. G. S. Kapur and A. S. Brar, J. Polym. Sci., A, 29, 479 (1991)

18. K. Kamide and K. Hisatani, Polym. J., 24, 1377 (1992).

19. K. Hisatani, K. Okajima, and K. Kamide, Polym. J., 27, 728 (1995).
20. K. Kamide, H. Yamazaki, K. Okajima, and K. Hikichi, Polym. J., 17, 1291 (1985).

21. K. Kamide, H. Yamazaki, K. Okajima, and K. Hikichi, Polym. J., 17, 1233 (1985).

22. K. Kamide, H. Yamazaki, and Y. Miyazaki, Polym. J., 18, 819 (1986).

23. K. Kamide, Y. Nakagawa, H. Yamazaki, and K. Hisatani, Polym. Prepr., Jpn., 42, 1495 (1993).

24. H. Yamazaki, Y. Miyazaki, M. Saito, and K. Kamide, Polym. J., 23, 725 (1991).

25. K. Hisatani, K. Okajima, and K. Kamide, Polym. J., 28, 99 (1996). 